Intensivmed 2010 · 47:148-148 DOI 10.1007/s00390-009-0116-2 Online publiziert: 4. März 2010

(c) Springer-Verlag 2010

\author{
S. Schwab \\ Neurologische Klinik, Universitätsklinikum Erlangen
}

\title{
Therapie des Schlaganfalls
} Nur die optimale Versorgung hilft

Dieses Themenheft zur Versorgung und intensivmedizinischen Therapie des Schlaganfalls fasst in 5 Beiträgen die wesentlichen Entwicklungen in der Behandlung des Schlaganfalls in den letzten Jahren zusammen. Wie Sie sicher wissen, ist der Schlaganfall mittlerweile in den industrialisierten Ländern die zweithäufigste Todesursache und die häufigste Ursache einer dauerhaften Behinderung. Wir müssen damit rechnen, dass ca. 200.00o Patienten in Deutschland jährlich einen akuten Schlaganfall erleiden. Glücklicherweise haben wir heute Behandlungskonzepte, die es uns ermöglichen, v. a. innerhalb der ersten Stunden einen signifikanten Nutzen für unsere Patienten mit Schlaganfall zu erreichen. Damit aber eine solche Therapie dem Patienten zugute kommen kann, ist es nach wie vor essenziell, dass Schlaganfallpatienten so schnell wie möglich in ein spezialisiertes Zentrum gelangen. Hier sind wir in Deutschland Vorreiter mit einer fast flächendeckenden Versorgung mit Stroke Units. Bevor aber der Patient auf der Stroke Unit behandelt wird, ist das rasche Ineinandergreifen von Notfall- und Akutversorgung auch in der Notfallambulanz und im Rettungsdienst notwendig.

Die Beiträge dieser Ausgabe von „Intensivmedizin und Notfallmedizin“ behandeln daher die optimale Versorgung in der Prähospitalphase über das weitere Management auf der Stroke Unit und auch spezielle intensivmedizinische Aspekte in der Behandlung von zerebraler Ischämie, intrakranieller Blutung oder Subarachnoidalblutung. Dieses Heft soll Ihnen eine Übersicht über den aktuellen Stand der klinischen Forschung und Versorgung geben und Ihnen die vielfältigen Aspekte auch in der interdisziplinären Zusammenarbeit von Notärzten, Neurologen, Internisten und anderen Berufsgruppen aufzeigen. Der Ihnen sicher bekannte Slogan „Time is brain“ kann gar nicht oft genug wiederholt werden und steht über allen Beiträgen. Ich hoffe, dass Sie auch als „fachfremder“ Leser erkennen, wie wichtig und wertvoll ein reibungsloses Ineinandergreifen von Prähospitalphase, Notaufnahme und Schlaganfallspezialstation in der Versorgung von Schlaganfallpatienten ist.

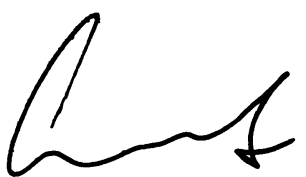

Stefan Schwab

\section{Korrespondenzadresse \\ Prof. Dr. S. Schwab \\ Neurologische Klinik, \\ Universitätsklinikum Erlangen \\ Schwabachanlage 6, 91054 Erlangen \\ Stefan.Schwab@uk-erlangen.de}

